\title{
Arbeiteremigration aus der Arabischen Republik Jemen in die Erdölstaaten der Arabischen Halbinsel
}

\section{Jemen - ein Entwicklungsland hat Arbeitskräfte-Mangel}

Arbeitslosigkeit und hohe Anteile von Arbeitskräften in der Landwirtschaft zählen zu den wichtigsten Merkmalen der meisten Entwicklungsländer; die Schaffung von neuen Arbeitsplätzen im sekundären und tertiären Wirtschaftssektor ist normalerweise mit großen Schwierigkeiten verbunden.

Die Arabische Republik Jemen zeigt eine für Entwicklungsländer recht ungewohnte Entwicklung. Noch vor 10 Jahren waren über $90 \%$ der Wohnbevölkerung in der Landwirtschaft tätig und konnten durch einen sehr arbeitsintensiven Terrassenfeldbau - vorwiegend auf der monsunbestrichenen westlichen Abdachung zum Roten Meer - eine weitgehend autarke Lebensmittelversorgung des Landes sicherstellen. 1971/72 wurden etwa für 20 Millionen US \$ landwirtschaftliche Importe getätigt, während die Exporte dem Lande 5 Millionen US \$ einbrachten. Unter dem Einfluß der raschen wirtschaftlichen Entwicklung, die im Anschluß an die «Erdölkrise» in den erdölfördernden Staaten der arabischen Halbinsel einsetzte, wuchs die temporäre Auswanderung von jemenitischen Arbeitskräften sprunghaft an. Bereits Mitte der 70er Jahre stellte sich im Jemen ein Arbeitskräftemangel ein, der die Behörden des Landes bewog, zeitweise durch Verweigerung der Abgabe von Reisepapieren eine zunehmende Auswanderung zu verhindern. Außerdem wanderten 40-60000 ausländische Arbeitskräfte (vorwiegend aus Ägypten, Pakistan, Indien) in das rohstoffarme Entwicklungsland Nordjemen ein.

Der 30\%ige Rückgang der in der Landwirtschaft tätigen männlichen Arbeitskräfte von über 1 Million (1970) auf unter 700000 (1980) konnte nur zu einem kleinen Teil durch Rationalisierungsmaßnahmen und Mechanisierung aufgefangen werden. Das Fehlen von rund einem Drittel der Familienväter führte einerseits zu einer beträchtlichen Mehrbelastung der im Lande verbleibenden Frauen und Kinder, anderseits zur extensiveren Pflege der früher sorgfältig unterhaltenen Terrassenflächen sowie zur teilweısen Aufgabe von landwirtschaftlichen Anbauflächen. Als wirtschaftliche Auswirkungen folgten ein massiver Rückgang in der Selbstversorgung, ein starkes Anwachsen der Importe und eine erschreckend hohe
Inflation auf Gütern, Mieten und auch Löhnen. Die Importe verfünfzigfachten sich innert weniger Jahre (1979/80 1500 Millionen US \$), während sich der Umfang der Exporte kaum veränderte (1979/80 7 Millionen US \$).

\section{Entwicklung, Ausmaß und Grundzüge der jemenitischen Arbeiterauswanderung}

\subsection{Vorbemerkungen zur Datenlage}

Zuverlässigkeit und Vollständigkeit von statistischen Angaben lassen in Entwicklungsländern häufig zu wünschen übrig. Einerseits rührt dies davon, daß die Kommunikationsmittel, Fachkräfte und finanziellen Mittel für die Durchführung und detaillierte Auswertung von Großerhebungen oft fehlen. Anderseits unterliegen die Behörden in Ländern mit ungenauer Datenlage oft der Versuchung, Datenlücken durch politische «Wunsch-Schätzungen» zu füllen. Im Falle der Arbeiteremigration im Mittleren Osten existieren verschiedene Beispiele, in denen einerseits die Angaben in den Auswanderungsstaaten künstlich hochgehalten, anderseits in den Einwanderungsstaaten absichtlich zu niedrig ausgewiesen werden. Am vertrauenswürdigsten sind oft Angaben, die direkt aus Zählungen, nicht politisch modifizierten Einwanderungsregistern und länderübergreifenden Vergleichsstudien stammen.

Auch in der Arabischen Republik Jemen weisen die Bevölkerungsangaben eine große Bandbreite auf. Seit der Durchführung der ersten Volkszählung des Landes im Jahre 1975 konnten jedoch zahlreiche Unklarheiten beseitigt werden.

Der Autor hatte die Gelegenheit, aufgrund eines bilateralen Vertrages zwischen der Schweiz (Direktion für Entwicklungszusammenarbeit, EDA) und Jemen (Central Planing Organization) ${ }^{1}$ ) die erste landweite Erhebung zu unterstützen und die wichtigsten Resultate zu analysieren.

1) Zusammen mit einer Gruppe von Geographen, Luftbildinterpreten und einem Architekten (W.Dubach, E. Egli, U. Geiser, R. Schoch)

Dr. H. Steffen, Bundesamt für Statistik, 3003 Bern 
Außer den Volkszählungsergebnissen werden in der vorliegenden Abhandlung hauptsächlich Angaben aus dem Bulletin of the Central Bank of Yemen, dem statistischen Jahrbuch und dem Paßbüro verwendet. Für detaillierte Quellenangaben sei auf ausführliche Abhandlungen verwiesen (siehe Literaturhinweise am Ende dieses Beitrages).

\subsection{Die langfristige Auswanderung}

Die Auswanderung von Bewohnern aus den dicht bevölkerten und landwirtschaftlich extrem genutzten Gebieten Jemens war bereits in früheren Jahrhunderten von Bedeutung. In historischen Berichten werden seit dem im Koran erwähnten Dammbruch von Saba (Ma'rib) in der Mitte des 4. Jahrhunderts nach Chr. verschiedene Wanderungswellen erwähnt, die vom Jemen durch die arabische Halbinsel nach den nordarabischen Ländern, besonders nach Mesopotamien, gerichtet waren.

Bis vor etwa 10 Jahren wanderten Tausende von Jemeniten auf der Suche nach Arbeit und Lebensunterhalt ins südlich gelegene, damalige britische Protektorat Aden aus. Eine beachtliche Zahl von Jemeniten gelangte auch über den Hafen von Aden in fernere Länder, bis in die Küstenstädte von England (Cardiff, Plymouth, London), Frankreich (Marseille) oder die Länder des Fernen Ostens (Indonesien, Thailand, Vietnam) und nach den Vereinigten Staaten von Amerikal (Detroit). Zahlreiche Jemeniten überquerten auch das Rote Meer und ließen sich in Äthiopien, Sudan, Djibouti, Kenya und anderen ostafrikanischen Ländern nieder. Mit Ausnahme der leicht erreichbaren Kolonie Aden wurden die Kontakte zwischen den Auslandjemeniten und ihren Angehörigen im Jemen oft während der generationenlangen Auslandabwesenheit unterbrochen. Die meisten dieser früheren Auswanderer verblieben auf Lebzeiten fern von ihrer Heimat und heirateten häufig Einheimische des Gastlandes.

\subsection{Die temporäre Auswanderung}

In den letzten 10 Jahren fand ein wesentlicher Wandel in der jemenitischen Auswanderung statt, indem die temporäre Auswanderung nach den benachbarten Erdölstaaten gewaltig an Bedeutung gewann. Diese Art der Auswanderung unterscheidet sich in mehreren Punkten von der früher üblichen langfristigen Auswanderung.

Ziel: Die temporäre Auswanderung ist vorwiegend auf die erdölfördernden arabischen Staaten ausgerichtet (Saudi-Arabien, Kuwait, Arabische Emirate).

Demographische Merkmale: Die Auswanderer sind fast ausschließlich männliche Jemeniten im arbeitsfähigen Alter, die ihre Familienangehörigen in den Dörfern im Jemen zurücklassen. Die Auswanderer sind zum größten Teil ungelernte Arbeitskräfte aus den ländlichen Gebieten, wobei die ärmsten wie auch die reichsten Bevölkerungsgruppen kaum vertreten sind. Schwierigkeiten bei der Erwerbung des Passes und bei der Abwicklung der Ausreiseformalitäten bei den ärmsten und ausreichende Verdienstmöglichkeiten im Lande bei den reichsten Bevölkerungsschichten mögen die Hauptgründe für diese Selektion sein. Beruflich qualifizierte Auslandjemeniten (Köche, Schneider, Maurer, Schreiner, Barbiere usw.) stammen zumeist aus den alten, traditionellen Auswanderungsgebieten des Landes.

Dauer der Abwesenheit: Die Auswanderer pendeln in relativ kurzen Abständen zwischen dem Gastland und ihren Herkunftsdörfern im Jemen hin und her. Einerseits richtet sich die Dauer der Auslandabwesenheit nach der Entfernung des Einsatzortes, anderseit besteht auch ein Unterschied zwischen ledigen und verheirateten Jemeniten. In Saudi-Arabien beträgt die Aufenthaltsdauer zur Zeit normalerweise 1-2 Jahre bei verheirateten und 2-4 Jahre bei noch unverheirateten Jemeniten.

Regionale Herkunft: Die Auswanderer rekrutieren sich aus sozusagen allen Gegenden des Landes, wobei jedoch drei Regionen durch besonders hohe Auswanderungsraten auffallen:

a) die traditionellen Auswanderungsgebiete im Süden des Landes entlang der Grenze zu Südjemen (Provinzen von $\mathrm{Al}$ Bayda', Ibb und Ta'izz);

b) die Gebirgsstücke westlich und nordwestlich der Landeshauptstadt; (Jabal Miswar, 'Iyāl Şurayḩ, 'Iyāl Yazid, Thulà, Ḩajjah usw.);

c) die Grenzgebiete zu Saudi-Arabien (im Norden und Nordosten des Landes).

\subsection{Die Entwicklung in den siebziger Jahren}

Seit dem Ende des Bürgerkrieges(1969) ist eine starke Rückwanderung von langfristigen Auslandaufenthaltern nach Jemen zu verzeichnen. Nachdem der Südjemen die Unabhängigkeit erlangte, verließen die meisten Nordjemeniten das ehemalige Britische Protektorat Aden und zogen in ihr Heimatland zurück. Auch aus Äthiopien und in jüngster Zeit aus Djibouti, Somalia und Sudan sind zahlreiche Auswanderer nach Jemen zurückgekehrt.

Gleichzeitig mit der Rückwanderung von langfristigen Auslandaufenthaltern setzte ein wachsender Strom von temporären Auswanderern nach denErdölstaaten ein. Im Gegensatz zu früheren Auswanderungsperioden verließen die Jemeniten den Jemen nicht, um einer Arbeitslosigkeit im eigenen Lande zu entfliehen, sondern weil die Nachfrage auf dem saudi-arabischen Arbeitsmarkt besonders groß und die angebotenen Löhne verlockend hoch waren. Die Anziehung aus dem Auslande wirkte so stark, daß im Jemen Mitte der 70er Jahre ein Mangel an Arbeitskräften auftrat und die Löhne für qualifizierte Facharbeiter (z. B. Steinmetze, Maurer, Leute mit 
Englischkenntnissen) und landwirtschaftliche Hilfskräfte stark anstiegen. Diese Entwicklung veranlaßte die jemenitische Regierung, die temporäre Auswanderung von weiteren Arbeitskräften ins Ausland zu verbieten und die Ausstellung von neuen Pässen drastisch einzuschränken. Jemeniten, die bereits vor 1976 in den Besitz eines Passes gelangt waren, durften weiterhin zwischen ihrem Einsatzort in SaudiArabien und dem Jemen hin- und herpendeln. Neue Pässe zur Ausreise wurden jedoch nur noch an Geschäftsleute, Studenten und Pilger ausgestellt. Bei der Einführung dieser neuen Regelung sah man lange Kolonnen von $\mathrm{Pa}$ bewerbern vor den Auswanderungsbüros stehen. Verschiedentlich sollen sich auch Auswanderer einen $\mathrm{Pa} \beta$ für die Pilgerreise nach Mekka verschafft haben und anschließend als Arbeiter in Saudi-Arabien verblieben sein. Eine unbestimmbare Zahl von Jemeniten dürfte auch auf dem Landwege ohne $\mathrm{Pa}$ ausgewandert sein. Gegenüber Angehörigen anderer Staaten haben die Jemeniten in Saudi-Arabien das Recht, ohne einen eigentlichen Arbeitsvertrag zu arbeiten. Dadurch können sie mühelos eine Stelle antreten und diese wiederum wechseln, sobald sie eine besser bezahlte Anstellung gefunden haben.

Zur Bekämpfung des Arbeitskräftemangels und zur Eindämmung der Lohnexplosion erlaubt die jemenitische Regierung die Beschäftigung von ausländischen Arbeitskräften aus Billiglohnländern. Die Einwanderungsbestimmungen sind relativ liberal. Im Bildungssektor arbeiten heutzutage mehrere hundert ägyptische, syrische und palästinensische Lehrkräfte.

In der nachstehenden Aufstellung wurde der Versuch unternommen, die oben beschriebene Entwicklung der Auswanderung jemenitischer Arbeitskräfte in Zahlen zu fassen. Ausgehend von den ziemlich gut gesicherten Volkszählungsangaben wurden die
Schätzungen für die früheren und nachfolgenden Jahre aufgrund zahlreicher Indikatoren (abgegebene Pässe, ausgestellte Visa, Beträge der Überweisungen, Anzahl der Schüler und Studenten im Ausland usw.), verschiedener Befragungen von Beamten in San' $\bar{a}$ ' und Besprechungen mit Auslandjemeniten aufgestellt. Dennoch muß betont werden, daß die Angaben nur dazu dienen sollen, eine Vorstellung über die Größenordnung der Aus- und Rückwanderungsströme zu vermitteln.

Nahezu vervierfacht hat sich die Zahl der temporären Auswanderer in den 70er Jahren. Arbeiteten 1970 erst etwa 140000 Jemeniten in den Erdölländern der Arabischen Halbinsel, so waren es am Ende des Jahrzehnts bereits über eine halbe Million. Den in relativen wie in absoluten Zahlen stärksten Anstieg zeigte die temporäre Auswanderung im Anschluß an die Vervierfachung der Rohölpreise durch die OPEC-Staaten (Herbst 1973). In den folgenden drei Jahren erhöhte sich die Zahl der jährlichen Auswanderer durchschnittlich um rund 70000 Personen. 1976/77 trat eine Stabilisierung auf dem Niveau von etwas über 500000 Auswanderern ein; dies ist auf folgende Faktoren zurückzuführen:

a) Die ungelernten jemenitischen Arbeitskräfte erfuhren eine zunehmende Konkurrenzierung durch die Einwanderung von asiatischen Arbeitskräften (aus Indien, Sri Lanka, Malaysia, Phillippinen, Südkorea), die sich durch berufliche Fähigkeiten, Ausdauer und bescheidenere Lohnforderungen auszeichnen.

b) Die Regierungen der Erdölförderländer haben ein gewisses Interesse daran, daß die Zahl der muslimischen Gastarbeiter nicht unkontrollierbar anwächst. Die übereinstimmende Sprache und Religion können innenpolitische Einmischungen erleichtern (z. B. Besetzung der Mo-

\section{Tab. 1 Schätzung der Zahl der kurzfristigen und langfristigen Auswanderer aus der Arabischen Republik Jemen}

zwischen 1970 und 1980

\begin{tabular}{|l|c|c|c|c|}
\hline Anfang & $\begin{array}{l}\text { Gesamtzahl } \\
\text { der Jemeniten } \\
\text { im Ausland }\end{array}$ & $\begin{array}{l}\text { Langfristige } \\
\text { Auswanderer } \\
\text { gesamthaft }\end{array}$ & $\begin{array}{l}\text { Kurzfristige } \\
\text { Auswanderer } \\
\text { gesamthaft }\end{array}$ & $\begin{array}{l}\text { Kurzfristige Aus- } \\
\text { wanderer, davon } \\
\text { erwerbstätig }\end{array}$ \\
\hline 1970 & 480000 & 330000 & 150000 & 140000 \\
1971 & 500000 & 320000 & 180000 & 170000 \\
1972 & 520000 & 310000 & 210000 & 200000 \\
1973 & 540000 & 290000 & 250000 & 300000 \\
1974 & 580000 & 270000 & 310000 & 365000 \\
1975 & 630000 & 250000 & 380000 & 450000 \\
1976 & 700000 & 230000 & 470000 & 580000 \\
1977 & 720000 & 220000 & 500000 & 510000 \\
1978 & 730000 & 210000 & 520000 & 520000 \\
1979 & 735000 & 205000 & 530000 & 540000 \\
1980 & 740000 & 200000 & & \\
\hline
\end{tabular}


schee von Mekka durch pakistanische Muslims). Die asiatischen Einwanderer zeigen in religiösen und politischen Angelegenheiten ein indifferenteres Verhalten.

c) Auch der starke Lohnanstieg im Jemen und die Regierungsmaßnahmen zur Beschränkung der Auswanderung mögen zur Stabilisierung beigetragen haben. Allerdings hat die zeitweise Unterbindung der Paßausgabe eine unerwünschte Selektion verursacht, da die Auswanderer, die vor 1976 in den Besitz eines Passes gelangt waren, weiterhin zwischen Jemen und dem Ausland hinund herreisen können.

Da jene Auswanderer meist beruflich besser qualifiziert sind als die zurückgebliebenen Jemeniten, könnten sie im Jemen besonders gut gebraucht werden. Hinzu kommt, daß beim Festhalten eines status quo regionale Unterschiede im Emigrantenanteil nicht mehr korrigiert werden können. Die einen Gebiete dürfen weiterhin eine hohe Auswanderungsrate beibehalten, während die anderen Gebiete, die vor 1976 noch kaum die Auswanderung kannten, nun weiterhin keine Auswanderer entsenden dürfen, obwohl regional ein Arbeitskräfteüberfluß besteht und jene Regionen ebenfalls an den Überweisungen von Auslandarbeitern interessiert wären.

\section{Wirtschaftliche und soziale Auswirkungen der temporären Auswanderung}

\subsection{Demographische Aspekte}

Zur Zeit wird das natürliche Bevölkerungswachstum im Jemen auf etwa 2,1\% pro Jahr geschätzt. Da etwa ein Drittel der verheirateten Männer ihren Lebensunterhalt getrennt von Frau und Familie im Ausland verdienten, muß angenommen werden, daß die Bevölkerungsentwicklung durch die temporäre Auswanderung maßgeblich beeinflußt wird. Wie jedoch die folgenden Beispiele zeigen, sind die Einflüsse zum Teil kontravers, so daß nicht eindeutig mit einer beschleunigenden oder verlangsamenden Wirkung auf das Bevölkerungswachstum gerechnet werden kann:

a) Die durch Auslandeinkommen verbesserte Kaufkraft ließ die Brautpreise in den siebziger Jahren stark ansteigen. Dadurch sind zahlreiche junge Männer gezwungen, vor der Heirat vorerst während einigen Jahren durch Arbeit im Ausland das für die Heirat notwendige Geld zu verdienen. Als Folge der temporären Auswanderung muß deshalb mit einer merklichen Erhöhung des mittleren Heiratsalters und des mittleren Alters der Mütter bei der Geburt des 1. Kindes gerechnet werden.

b) Da die temporären Auswanderer in regelmäßigen Abständen nach Jemen zurückkehren, ist es kaum wahrscheinlich, daß die zeitweilige Tren- nung der Ehegatten einen großen Einfluß auf die jährliche Zahl der Geburten hat.

c) Durch Erhalt von Geldsendungen von ihren im Ausland arbeitenden Ehegatten haben die jemenitischen Ehefrauen vermehrt die Möglichkeit, die Ernährung mit gekaufter Trockenmilch einzusetzen und rascher das Stillen von Kleinkindern abzubrechen. In Gebieten mit schlechtem Trinkwasser können dadurch die Infektionsgefahr und die Kindersterblichkeit zunehmen. Außerdem setzt bei den nicht mehr stillenden Müttern rascher die Monatsregel wieder ein, wodurch Schwangerschaften in kürzeren Abschnitten auftreten können.

d) Eine nicht zu unterschätzende Bedeutung kommt den wechselnden Ansichten über die ideale Kinderzahl zu. Im allgemeinen ist bei steigendem Lebensstandard ein Sinken der gewünschten Kinderzahl zu beobachten.

\subsection{Soziale Auswirkungen}

Die Auswanderung erlaubt den jungen Männern, aus den sozialen und stammespolitischen Strukturen auszubrechen und ein unabhängiges Leben aufzubauen. Im Ausland lernen die Emigranten Anschauungen kennen, die eher von wirtschaftlichen Faktoren als von traditionellen Werten bestimmt werden. Dadurch können kulturelle und soziale Wertungen oder die Rolle und Rechte von Stammesführern und Shaykh's in Frage gestellt werden.

Anderseits können die Auswanderer breite Erfahrungen und berufliche Fähigkeiten nach Jemen zurückbringen und damit dem Lande wertvolle neue Entwicklungsimpulse verleihen. Insbesondere im Bereich der Hygiene, des Gesundheitswesens, der Schule, der Landwirtschaft und der genossenschaftlichen Selbsthilfeorganisation sind manche Neuerungen auf Initiative von Emigranten in die Wege geleitet worden.

\subsection{Private Transferzahlungen}

Für die Wirtschaft ist die Auswanderung besonders wegen der Rücksendung von Ersparnissen der Auslandjemeniten an ihre Familienangehörigen von Bedeutung.

\section{Traditionelle Landwirtschaft}

In den 70er Jahren ging die landwirtschaftliche Anbaufläche im Jemen infolge Arbeitskräftemangel und konkurrenzierende Nahrungsmittelimporte um $30 \%$ zurück.

a) Terrassierter Steilhang am Jabel Sabir, bepflanzt mit QatKulturen.

b) Bauer verkleinert die Erdschollen mit einem Holzhammer, um das Austrocknen des Bodens zu verhindern, Region Al Hujariyah.

c) Bauern in der Region von Dhamar beim Pflügen.

(Aufnahmen: H. Steffen, Dez. 1972, Jan. 1973, März 1976.) 


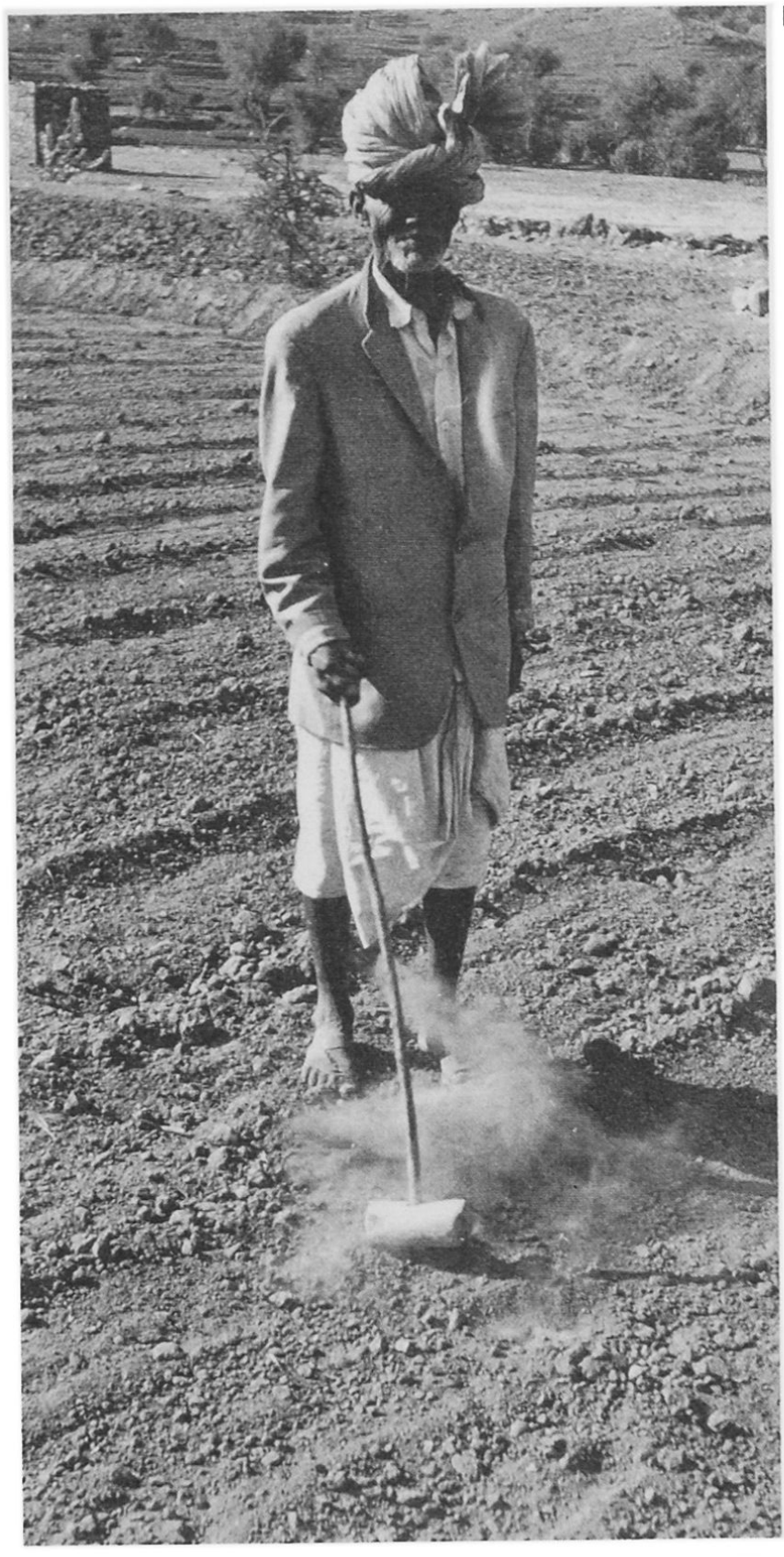

c)
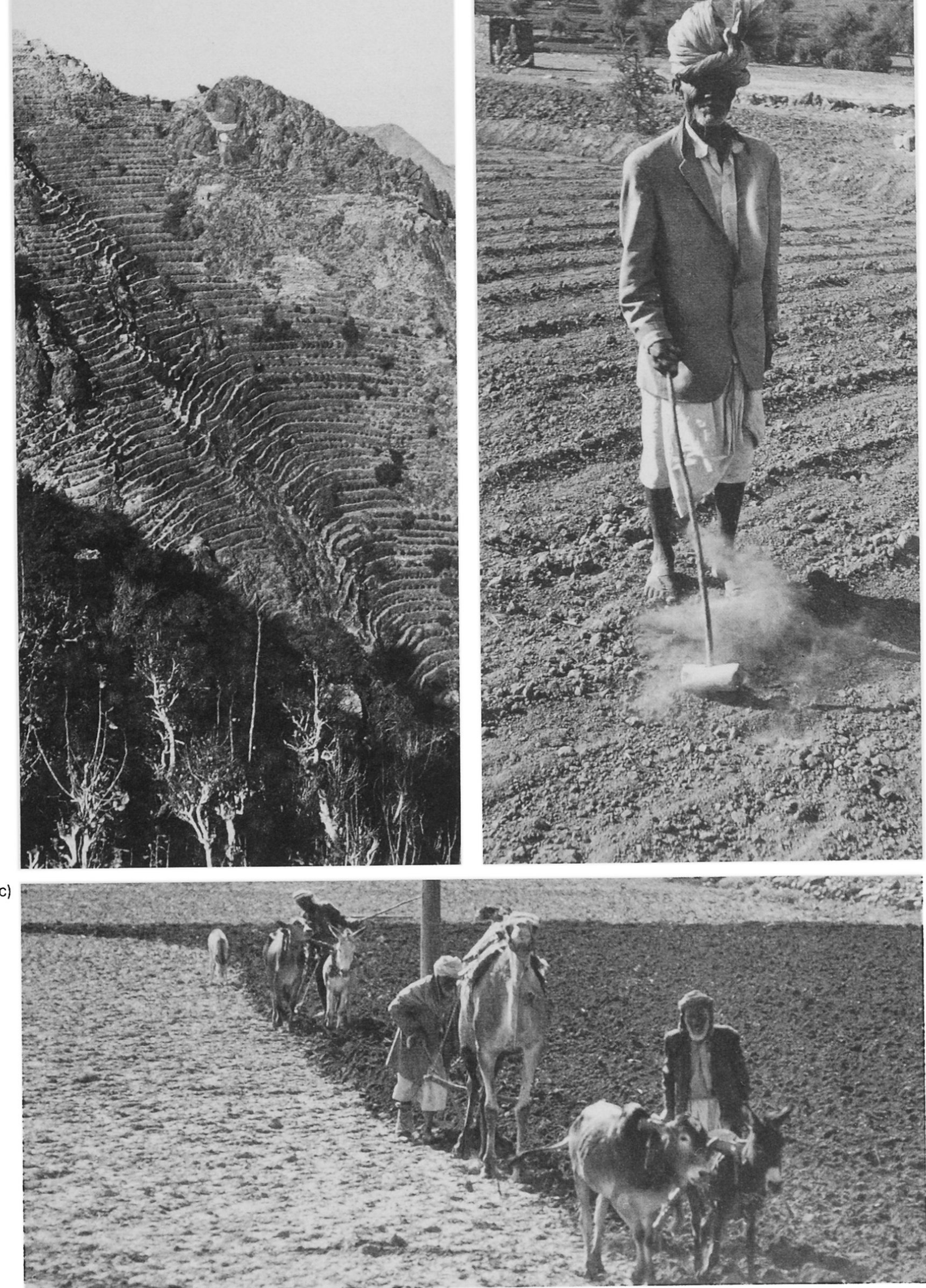
Tab. 2 Transferzahlungen nach Jemen zwischen 1970/1 und 1979/80 sowie Prokopfüberweisungen der temporären Auslandjemeniten (Berechnungsannahme 25 Arbeitstage pro Monat)

\begin{tabular}{|c|c|c|c|c|c|}
\hline Periode & $\begin{array}{l}\text { Private Transfer- } \\
\text { zahlungen nach Jemen } \\
\text { (in Yemen Rial = 0,45 US\$) }\end{array}$ & $\begin{array}{l}\text { Geschätzte Zahl } \\
\text { der temporären } \\
\text { Auslandarbeiter } \\
\text { (Stand Februar) }\end{array}$ & $\begin{array}{l}\text { Durchschnittlich } \\
\text { pro Kopf der tem } \\
\text { Auslandarbeiter } \\
\text { jährlich }\end{array}$ & $\begin{array}{l}\text { ëberweis } \\
\text { porären } \\
\text { monatlich }\end{array}$ & gen \\
\hline $\begin{array}{l}1970 / 71 \\
1971 / 72 \\
1972 / 73 \\
1973 / 74 \\
1974 / 75 \\
1975 / 76 \\
1976 / 77 \\
1977 / 78 \\
1978 / 79 \\
1979 / 80\end{array}$ & $\begin{array}{r}237000000 \\
414000000 \\
452000000 \\
595000000 \\
1013000000 \\
2363000000 \\
4561000000 \\
6351000000 \\
6404000000 \\
6118000000\end{array}$ & $\begin{array}{l}170000 \\
200000 \\
240000 \\
300000 \\
365000 \\
450000 \\
480000 \\
500000 \\
510000 \\
520000\end{array}$ & $\begin{array}{r}1400 \\
2100 \\
1900 \\
2000 \\
2800 \\
5300 \\
9500 \\
12700 \\
12600 \\
11800\end{array}$ & $\begin{array}{r}116 \\
173 \\
157 \\
165 \\
231 \\
438 \\
792 \\
1059 \\
1046 \\
980\end{array}$ & $\begin{array}{r}4,60 \\
6,90 \\
6,30 \\
6,60 \\
9,30 \\
17,50 \\
31,70 \\
42,30 \\
41,90 \\
39,20\end{array}$ \\
\hline
\end{tabular}

Tab. 2 zeigt, daß die privaten Transferzahlungen zwischen 1973/74 und 1977/78 sprunghaft gewachsen sind: aufgrund einer fast jährlichen Verdoppelung stiegen die Auslandeinkünfte im Jemen innert 4 Jahren von 0,6 auf über 6 Milliarden YR an.

Bemerkenswert ist, daß der Verzehnfachung der Transferzahlungen «nur» eine Verdoppelung der Zahl der Erwerbstätigen gegenübersteht (1972/73: 240000, 1976/77: 480000). Das raschere Wachstum der Transferzahlungen ist $\mathrm{zu}$ einem wichtigen Teil auf die hohe Inflation in den Erdölländern und die Lohnerhöhungen zurückzuführen. Jemeniten, die in Saudi-Arabien arbeiten, berichten, daß der Taglohn für ungelernte Hilfskräfte Anfang 1973 etwa SR 10.- bis SR 20.- betrug. Anfang 1976 stiegen sie auf etwa SR50.- und Ende 1977 erreichten sie SR80.bis $100 .-(1 \mathrm{SR}=0,60 \mathrm{US} \$)$.

Tab. 2 zeigt überdies, daß die privaten Transferzahlungen vom Ausland nach Jemen seit 1970/71 (237 Mio YR) massiv angestiegen sind und 1979/80 den phantastischen Betrag von YR 6118 Mio erreicht haben. Nach Auskunft der Jemenitischen Zentralbank sollen in den von ihr veröffentlichten Angaben sämtliche Wechselgeschäfte von Saudi Rial und anderen Fremdwährungen in jemenitische Rial enthalten sein, gleichgültig ob das Geld im Ausland oder Jemen gewechselt oder nach Jemen überwiesen wurde. Der Großteil der privaten Transferzahlungen stammte von temporären jemenitischen Auslandarbeitern, die die Ersprarnisse an ihre Angehörigen überwiesen oder als Bargeld persönlich zurückbrachten.

Vergleicht man die Prokopfüberweisung mit den Löhnen in Saudi-Arabien, ergibt sich eine durchschnittliche Überweisungsquote von etwa $30-50 \%$ des Lohnes. Darin nicht inbegriffen sind die Warensendungen aus Saudi-Arabien nach Jemen, die ebenfalls einen beachtlichen Umfang erlangt haben:
Transistorradios, TV-Geräte, Wolldecken, Matratzen, Teppiche, Stoffe, Kleider, Metallkoffer, Geschirr, Schmuck, Parfums usw.

Über die Gründe des Rückganges der Transferzahlungen zwischen 1978 und 1980 lassen sich nur Vermutungen aufstellen. Einen Einfluß könnten folgende Faktoren gehabt haben:

- Vermehrter Einkauf von Edelmetallen, Silber-, Goldschmuck und Waren, um der raschen Geldentwertung zu begegnen

- leichte Reallohnabnahmen in Saudi-Arabien infolge Konkurrenzierung durch asiatische Arbeitskräfte

- geringe Ersparnisse infolge kürzerer Auslandaufenthalte, höherer Reisekosten und gestiegener Lebenskosten in Saudi-Arabien

\subsection{Verwendung der privaten Transferzahlungen im Jemen}

Tab. 3 zeigt das starke Wachstum von BruttoInlandprodukt und Brutto-Nationalprodukt in den 70er Jahren. Deutlich tritt die Bedeutung der privaten Transferzahlungen hervor, die etwa 40-50\% zum Brutto-Nationalprodukt beitrugen.

Zwar fehlen landweite Untersuchungen über die detaillierte Verwendung der im Ausland verdienten Gelder, aber es können doch die wichtigsten Verwendungsbereiche angegeben werden.

\section{a) Anschaffung von Konsumgütern}

Ein wichtiger Teil des im Ausland verdienten Geldes wird verwendet, um Nahrungsmittel zu kaufen, die nach der Abwanderung von Arbeitskräften nicht mehr durch die jemenitischen Familien selbst hergestellt werden können: Getreide, Früchte, Gemüse, Fleisch. Hinzu kommen Produkte, die nicht selbst im Lande produziert werden und früher nur in sehr 


\begin{tabular}{|l|r|r|c|}
\hline & $1970 / 71$ & $1975 / 76$ & $1979 / 80$ \\
\cline { 2 - 4 } Brutto-Inlandprodukt (in YR) & 330 Mio & 2000 Mio & 7000 Mio \\
\hline $\begin{array}{l}\text { Saldo der privaten Transferzahlungen } \\
\text { Saldo der öffentlichen Transferzahlungen } \\
\text { Dienstleistungen }\end{array}$ & $\begin{array}{r}210 \text { Mio } \\
-\end{array}$ & $\begin{array}{r}2060 \text { Mio } \\
-200 \text { Mio } \\
+170 \text { Mio }\end{array}$ & $\begin{array}{r}4940 \text { Mio } \\
-980 \text { Mio } \\
-\end{array}$ \\
\hline Brutto-Nationalprodukt (in YR) & 540 Mio & 4030 Mio & 10960 Mio \\
\hline $\begin{array}{l}\text { DE FACTO Bevölkerung am Jahresanfang } \\
\text { Zahl der temporären Auslandjemeniten }\end{array}$ & $\begin{array}{r}4280000 \\
180000\end{array}$ & $\begin{array}{r}4760000 \\
500000\end{array}$ & $\begin{array}{r}5210000 \\
540000\end{array}$ \\
\hline DE JURE Bevölkerung & 4460000 & 5230000 & 5750000 \\
\hline $\begin{array}{l}\text { Brutto-Inlandprodukt } \\
\text { pro Kopf der Wohnbevölkerung (in YR) }\end{array}$ & 77 & 420 & 1344 \\
\hline $\begin{array}{l}\text { Brutto-Nationalprodukt } \\
\text { pro Kopf der DE JURE Bevölkerung (in YR) }\end{array}$ & 121 & 770 & 1900 \\
\hline
\end{tabular}

geringem Umfange gekauft werden konnten (Zukker, Gewürze, Reis). Zu den eigentlichen LuxusKonsumgütern sind Parfums, Qat, wertvolle Stoffe und Kleider zu zählen.

\section{b) Bezahlung des Brautpreises}

Der Brautpreis, der an die Eltern der Braut entrichtet wird, stellt eine wichtige finanzielle Absicherung der Braut im Scheidungsfalle dar. Gleichzeitig kann dieser Betrag als Altersvorsorge der Brauteltern betrachtet werden. In Gebieten mit hoher Anzahl von Auslandarbeitern sind die Brautpreise in den letzten Jahren ins Unermeßliche gestiegen und betragen vielerorts zwischen YR 20000 und YR 50000. Nach Einschränkung der Auswanderung wurde im Juni 1976 durch den Staatspräsidenten eine Verordnung erlassen, die den Höchstpreis bei Heirat auf YR 3000 bis YR 6000 festlegte. Trotz hoher Strafen bei Forderung von höheren Brautpreisen blieb das Gesetz auf dem Lande praktisch wirkungslos.

\section{c) Investitionen}

Die Transferzahlungen können einem Entwicklungsland wichtige Impulse zur Mechanisierung der Landwirtschaft und Industrialisierung geben, sofern sie zur Finanzierung von Investitionen verwendet werden. Im Jemen ist jedoch festzustellen, daß der überwiegende Teil der Emigrantengelder für den Kauf von Konsumgütern verwendet und den Investitionen noch relativ wenig Bedeutung geschenkt wird. Am ehesten wird Geld für folgende «produktive» Anschaffungen ausgegeben:
- Hausbau, Bodenspekulation,

- Kauf von Fahrzeugen für den Taxidienst und Transporte,

- Kauf von Pumpen für die Bewässerung und die Trinkwasserversorgung,

- Kauf von Traktoren, Bau von Brunnen, Kanalanlagen und Installationen von Generatoren zur Elektrizitätsversorgung,

- Investitionen im Kleingewerbe: Einrichtung von Geschäften des Detailhandels, Werkstätten usw.

\subsection{Handelsbilanz und Importabhängigkeit}

Der schnelle Anstieg der Kaufkraft in den Privathaushaltungen hatte eine spürbare Zunahme der wirtschaftlichen Aktivität im Jemen zur Folge. Aber weder Landwirtschaft noch Handel und Industrie waren in der Lage, der stark steigenden Nachfrage nach Lebensmitteln und anderen Gütern nachzukommen. Als Folge trat in den 70er Jahren eine starke Inflation ein, die nach den offiziellen, eher niedrig gehaltenen Angaben jährlich $30 \%$ bis $40 \%$ beträgt, bei verschiedenen Gütern (Erdölprodukten, Immobilien, Landpreisen), Mieten und Löhnen sogar $50 \%$ pro Jahr überschritt.

Zur Bekämpfung der Inflation sah die Regierung 1977 folgende Maßnahmen vor:

a) Einschränkung der temporären Auswanderung von Arbeitskräften

b) Erhöhung der Importe zur Befriedigung der hohen Nachfrage nach Konsumgütern 
Tab. 4 Exporte, Importe, Handel-, Zahlungs- und Ertragsbilanz in der Arabischen Republik Jemen zwischen 1970/1 und 1979/80 (in Millionen YR)

\begin{tabular}{|l|r|r|r|r|r|r|r|}
\hline $\begin{array}{l}\text { Verhältnis } \\
\text { Export/Import } \\
\text { (in \%) }\end{array}$ & $1971 / 72$ & $1972 / 73$ & $1974 / 75$ & $1976 / 77$ & $1977 / 78$ & $1978 / 79$ & $1979 / 80$ \\
\cline { 2 - 8 } & $12,5 \%$ & $16,4 \%$ & $5,4 \%$ & $2,5 \%$ & $0,8 \%$ & $0,2 \%$ & $0,5 \%$ \\
\hline $\begin{array}{l}\text { Exporte } \\
\text { Importe }\end{array}$ & 14 & 25 & $\begin{array}{r}53 \\
411\end{array}$ & $\begin{array}{r}84 \\
2318\end{array}$ & $\begin{array}{r}32 \\
4135\end{array}$ & $\begin{array}{r}13 \\
6243\end{array}$ & $\begin{array}{r}32 \\
6958\end{array}$ \\
\hline $\begin{array}{l}\text { Handelsbilanz } \\
\text { Zahlungsbilanz }\end{array}$ & -161 & -386 & -928 & -3234 & -4103 & -6230 & -6926 \\
+212 & +480 & +859 & 4457 & 5574 & 6020 & 5445 \\
\hline Ertragsbilanz & 51 & 94 & -69 & 1223 & 1471 & -211 & -1480 \\
\hline
\end{tabular}

c) Erhöhung der Steuern zur teilweisen Abschöpfung der Kaufkraft

Die Förderung der Importe dürfte eine gefährliche Methode zur Bekämpfung der Teuerung sein. Wie Tab. 4 zeigt, sind die Importe ohnehin schon gewaltig angestiegen; zwischen 1972/73 und 1978/79 haben sie sich verfünfzehnfacht. Demgegenüber wiesen die Exporte ein viel geringeres Wachstum auf und erlitten seit 1976/77 sogar einen Rückgang. Vermochten 1970/71 noch etwa 8\% der Importausgaben durch Ausfuhren gedeckt werden, machte deren Anteil 1979/80 nur noch 0,5\% der Importe aus.

Während einigen Jahren konnte die stark defizitäre Handelsbilanz noch durch die privaten Transferzahlungen ausgeglichen werden, die mit Abstand den wichtigsten Beitrag an die stark positive Bilanz im Zahlungsverkehr leisten. Doch seit 1978/79 reichen auch jene Überschüsse nicht mehr aus, um die weiterhin stark steigenden Handelsbilanzdefizite zu decken.

Durch die Erhöhung der Importzölle könnten sowohl die schwerwiegende Konkurrenzierung der im Lande erzeugten landwirtschaftlichen Güter reduziert, als auch der Aufbau von landeseigenen Produktionsstätten (Gewerbe, Kleinindustrie) gefördert werden. Ebenfalls sollte es der Regierung damit im Laufe der Zeit möglich sein, einen größeren Teil ihres bescheidenen Budgets durch eigene Mittel zu decken. Allerdings stößt die Realisierung von Zollund Steuererhöhungen auf fast unlösbare Probleme, weil kaum geschultes Personal in genügender Zahl für die Durchführung solcher Maßnahmen verfügbar gemacht werden kann.

\section{Auswirkungen der Arbeiteremigration auf die Landwirtschaft}

Die wichtigsten Auswirkungen der Arbeiteremigration auf die Landwirtschaft lassen sich wie folgt zusammenfassen:
- massiver Rückgang der landwirtschaftlich genutzten Kulturfläche, insbesondere im Getreideanbau

- stärkere Beteiligung von Frauen und Kindern in der Landwirtschaft

- Ansätze zur Rationalisierung und Mechanisierung, insbesondere im Bereich der künstlichen Bewässerung und beim Pflügen

- erhöhte Nachfrage nach Importgütern und Abbau des Selbstversorgungsgrades

- Verteuerung von landwirtschaftlichen Entwicklungsprojekten aufgrund massiver Preis- und Lohnsteigerungen

- Bereitschaft gewisser Landbesitzer, ihr Land zu günstigeren Pachtbedingungen abzugeben, um der Abwanderung von Pächtern entgegenzutreten.

Anschließend sollen einige Entwicklungen genauer belegt und deren positive und negative Seiten analysiert werden.

\subsection{Veränderungen in der Flächennutzung und Pro- duktion}

In der Landwirtschaft betrug 1979/80 der Tagesansatz für Arbeitskräfte YR 40 bis YR 50; dies entspricht etwa dem zwölffachen Betrag von 1972/73. Im Vergleich dazu stiegen der allgemeine Preisindex und die für landwirtschaftliche Produkte bezahlten Preise «nur» um das 4,5fache.

Landweit ist in der zweiten Hälfte der 70er Jahre die Getreideanbaufläche von schätzungsweise 1,2 Millionen ha auf unter 900000 ha zurückgegangen. Da die aufgegebenen Getreideanbauflächen kaum für ertragreichere Anbauprodukte Verwendung fanden, wird geschätzt, daß innert weniger Jahre etwa $30 \%$ des Kulturlandes brachgelegt worden sind. Die meisten der aufgegebenen Gebiete liegen im gebirgigen Teil des Landes - in Gebieten, die früher in aufwendiger Arbeit zu einer Terrassenlandschaft umgewandelt und alljährlich unter großem Aufwand vor 
Tab. 8 Wert der wichtigsten Nahrungsmittel-Importe zwischen 1971/72 und 1979/80 (in Millionen YR)

\begin{tabular}{|l|r|r|r|r|r|}
\hline & $1971 / 72$ & $1973 / 74$ & $1975 / 76$ & $1977 / 78$ & $1979 / 80$ \\
\cline { 2 - 5 } Getreide & 45 & 154 & 250 & 235 & 400 \\
Zucker & 21 & 102 & 249 & 132 & 253 \\
Gemüse & 9 & 32 & 70 & 221 & 483 \\
Kaffee, Tee, Gewürze & 6 & 23 & 48 & 78 & 73 \\
Milchprodukte, Eier & 2 & 15 & 35 & 123 & 341 \\
Fleisch & 0,1 & 0,6 & 4 & 123 & 223 \\
Verschiedenes & 8 & 38 & 86 & 70 & 122 \\
\hline Nahrungsmittel-Importe & 91 & 365 & 742 & 982 & 1895 \\
\hline Übrige Importe & 84 & 745 & 1797 & 3153 & 5063 \\
\hline \%-Anteil der Nahrungsmittel & $52 \%$ & $32 \%$ & $29 \%$ & $24 \%$ & $27 \%$ \\
\hline
\end{tabular}

der Erosion bewahrt worden sind. Da der Zerfall von Stützmauern und Terrassenbändern nach Aufgabe der landwirtschaftlichen Nutzung rasch fortschreitet, ist kaum damit zu rechnen, daß solche Gebiete zu einem späteren Zeitpunkt - z. B. unter veränderten wirtschaftlichen Bedingungen in den Erdölländern - jemals wieder landwirtschaftlich genutzt werden können (siehe Fotos).

\subsection{Verstärkte Beteiligung der Frauen}

Durch verstärkte Beteiligung von Frauen und Familienangehörigen in der Landwirtschaft konnte dem Mangel an männlichen Arbeitskräften am wirksamsten begegnet werden. Das Säen, Verkleinern der Erdschollen mit einem Holzhammer, Ernten, Hüten des Viehs usw. muß oft durch die Frauen und älteren Kinder erledigt werden, weil die erwachsenen Männer im Ausland arbeiten. Selbst die Pflugarbeit früher als ausschließliche Männerarbeit betrachtet ist in gewissen Regionen auf die Frauen übertragen worden.

Die Erledigung von zusätzlichen landwirtschaftlichen Arbeiten stellt für manche Mütter eine schwere Zusatzbelastung dar. Bereits dahin war das Tagesprogramm der Frauen durch Holzsammeln, Wassertragen, Spinn-, Web-, Näharbeiten, Getreidemahlen, Brotbacken, Kochen, Waschen, Putzen, Kinderpflege usw. stark ausgefüllt. Es ist deshalb nicht verwunderlich, daß manche Mütter erklären, daß sie zum Stillen keine Zeit mehr haben und auf Kauf von Kindernahrung angewiesen sind.

Die Realisierung von Projekten, um die bisherige Arbeitslast der jemenitischen Frauen zu erleichtern, sollte als vordringliches Ziel in die Entwicklungsprogramme von Regierung und Hilfsorganisationen aufgenommen werden. Durch die Errichtung von Dorfbrunnen könnte beispielsweise vielerorts der mühsame Marsch der Frauen an entfernte Quellen eingespart werden.

\subsection{Mechanisierung}

Die Mechanisierung der jemenitischen Landwirtschaft erfolgte in einem für Entwicklungsländer eher ungewöhlich raschen Tempo. Zwischen 1971 und 1978 erhöhte sich die Zahl der Traktoren im Lande von 100 auf 5000 und die Zahl der Dieselpumpen für die Bewässerung von 1200 auf 40000 .

Im allgemeinen sind die Bauern daran interessiert, neue Methoden anzuwenden, die künstlich bewässerten Anbauflächen durch Dieselpumpen auszuweiten, mit neuem Saatgut, Düngemittel und Pflanzenschutzmittel den Ertrag zu steigern. Allerdings muß leider häufig ein Mangel an Grundkenntnissen festgestellt werden. Zahlreiche Traktoren sind für die gebirgigen Verhältnisse zur groß und zu schwer, weil sie vorwiegend aus Prestigegründen mit möglichst hoher PS-Zahl angeschafft wurden. Verschiedentlich wird zu tief gepflügt, wodurch die Bodenabschwemmung und Bodenausblasung begünstigt werden. In den mechanischen Werkstätten sammeln sich Traktorenwracks, die durch unsachgemäßen Unterhalt Motorschäden erlitten haben. Durch solche Pannen ist zu erklären, daß bis dahin durch die Mechanisierung nur eine relativ kleine Zahl von Arbeitskräften eingespart werden konnte und der Anbau- und Produktionsrückgang nicht im gewünschten Maße verhindert werden konnte.

Vielerorts bildet auch das gebirgige Relief ein kaum überwindbares Problem für eine verbreitete Verwendung von Traktoren und größeren Maschinen. Weiterhin wird es kaum möglich sein, das Ochsengespann und den leichten Holzpflug auf den unzähligen schmalen Terrassenbändern auf der Westabdachung des Landes zu ersetzen. 


\section{Abschließende Bemerkungen}

Wie die vorangegangenen Ausführungen zeigten, bringt die Nachbarschaft zu finanzstarken Erdölstaaten der Arabischen Republik Jemen nicht nur Vorteile. Wohl resultieren aus den privaten Transferzahlungen der Auslandjemeniten zusätzliche Einnahmen in Milliardenhöhe, die die hohen Defizite des Außenhandels annähernd auszugleichen vermögen. Außerdem konnte das Entwicklungsland Jemen dadurch seit Mitte der 70er Jahre das Problem der Arbeitslosigkeit beheben. Anderseits mußten beachtliche Opfer erbracht werden. In der traditionellen Landwirtschaft und beim Häuserbau können nicht mehr die herkömmlichen arbeitsintensiven Methoden angewandt werden. Durch den Rückgang der Inlandproduktion und Aufblähung der Importe geriet das Land in eine gefährliche Auslandabhängigkeit. Eine unerwartete Rezession in den benachbarten Erdölländern müßte für die Arabische Republik Jemen verhängnisvolle Folgen haben.

Die Regierung im Jemen ist sich der Gefahren einer übertriebenen Arbeiteremigration bewußt. Doch sie hat relativ wenig Spielraum, um Maßnahmen zu treffen, die die negativen Einflüsse der Auswanderung reduzieren, ohne die positiven Auswirkungen aufzuheben. Zusätzlich stellen die noch geringe Effizienz und das schwache Durchsetzungsvermögen der jungen Administration wichtige Grenzen im politischen Handlungsvermögen dar.

Maßnahmen, die in den 80er Jahren durchgesetzt werden sollten, betreffen folgende Bereiche:

a) Gezielte, regionale und berufsspezifische Kontrolle der Arbeiterauswanderung;

b) Unterstützung von Bestrebungen zur Mechanisierung, Rationalisierung in der Landwirtschaft, um auch bei weiterhin leicht rückläufigem Arbeitskräftebestand eine Reduktion von Nutzfläche und Produktion zu verhindern. Staatliche Prüfung von Geräten und Maschinen, um die Einführung angepaßter Technologien sicherzustellen. Förderung der wissenschaftlichen Forschungen und Betriebsberatung in der Landwirtschaft;

c) Erhöhung der Importzölle auf Luxusgütern und Nahrungsmitteln, die die Inlandproduktion gefährden.

Wie die Zahlen von 1979 und 1980 zeigen, ist mit einer gewissen Stabilisierung in der Zahl der jemenitischen Auslandarbeiter wie auch der privaten Transferzahlungen zu rechnen. Es ist zu hoffen, daß die jemenitische Regierung die 80er Jahre für die Lösung der landesinternen Probleme zu nutzen versteht, die während der überraschenden Wirtschaftsexpansion in den 70er Jahren nicht unter Kontrolle gebracht werden konnten.

\section{Literaturhinweise}

1) ALLMANN et HILL: «Fertility, Morality, Migration and Family Planning in the Yemen Arab Republic», The Population Council, Amman 1977, p. $10-15$.

ATTAR, M.S.: «Le sous-développement économique et social au Yemen», Alger, 1964, p. 59-64.

CENTRAL BANK OF YEMEN: «Financial Statistical Bulletin» San'a', (Ausgaben 1-8)

CENTRAL PLANNING ORGANIZATION: «At Ta'ad al masakin wa as sukan, an nat'aj al awliyah», San'a', 1976.

- Statistcal Yearbook of the Yemen Arab Republic, San'a' (verschiedene Jahrgänge)

DAVID C.\& P.: «Preliminary Results of a Survey to Determine Women's Attitudes to Family Planning», San'a', 1978/3.

MYNTTI CYNTHIA: «Demographic Surveys carried out in the Yemen Arab Republic: Breastfeeding and Fertility», Mimeographed Paper, San'a', 1978/1.

- «Demographic Surveys carried out in the Yemen Arab Republic: Contraception in Yemen», San'a', 1978/2.

- «Demographic Surveys carried out in the Yemen Arab Republic: Emigration and Fertility», San'a', 1978/3.

GREEN J.W.: «Local Initiative in Yemen: Exploratory Studies of Four Local Development Associations», Mimeographed, 1975, Washington.

GHALEB, ANAM MOHAMED: «Government Organizations as a Barrier to Economic Development in the Yemen Arab Republic», NIPA, San'a', Bochum, 1979.

STEFFEN HANS,GEISER URS, DUBACH WERNER et al.: «Final Report of the Airphoto Interpretation Project of the Swiss Technical Co-opération Service», Berne, 1978, cf p. I/53.

STEFFEN HANS: «Population Geography of the Yemen Arab Republic, the Major findings of the Population and Housing Census of February 1975 and of Supplementary Demographic and Cartographic Surveys», Beiheft Nr.39 zum Tübinger Atlas des Vorderen Orients, L. Reichert, Wiesbaden, 1979, p. 56-60. 